\title{
Estudio médico-legal del homicidio en la provincia de Sevilla (2004-2007). Especial referencia a los homicidios de mujeres en el contexto de violencia de género.
}

\author{
Medico-legal study of the homicide in the province of \\ Seville (2004-07). Special reference to the female victims \\ inside the gender violence.
}

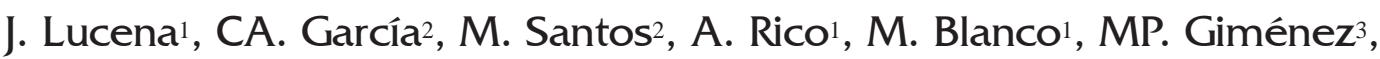
R. Marín ${ }^{1}$ y E. Barrero ${ }^{1}$

\section{RESUMEN}

Este estudio retrospectivo analiza los 57 homicidios ocurridos en la provincia de Sevilla durante un periodo de cuatro años (2004-2007).

Se han estudiado diversas variables relacionadas con los mismos obteniéndose los siguientes resultados: la tasa media de homicidios ha sido de $\mathbf{0 . 7 7}$ por $\mathbf{1 0 0 . 0 0 0}$ habitantes; el arma blanca es el método más utilizado seguido por el arma de fuego, contusiones y asfixia mecánica; las víctimas son en su mayoría varones (relación hombre/mujer 2:I) con una edad media de $46 \pm 21.2$ años; el mayor número de casos se registra en fines de semana (sábado y domingo) y durante el mes de octubre. El análisis toxicológico de las víctimas detectó etanol en el $47.4 \%$, benzodiacepinas en el $14 \%$, cocaína en el $12.3 \%$, opiáceos en el $10.5 \%$ y tetrahidrocannabinol en el $8.8 \%$.

Se ha hecho una valoración más detallada de los casos de homicidio dentro del contexto de violencia de género dada su alta incidencia en nuestro país. En 18 de 19 casos, las mujeres murieron a manos de su pareja, ex-pareja o familiar en primer grado.

Los resultados han sido comparados con estudios similares realizados tanto en el ámbito nacional como internacional.

Palabras clave: homicidio, patología forense, violencia de género.

Cuad Med Forense 2008; 14(51):35-46

\section{ABSTRACT}

This retrospective study analyzes the 57 homicides occurred in the province of Seville in a period of four years (2004-2007).

Some variables related with the homicides have been studied with the following results: the mean homicide rate was 0.77 per 100.000 inhabitants; sharp injury was the most frequent mechanism followed by shot gun, contusions and mechanical asphyxia; victims are in major part of cases males (male/female ratio 2:I) with a mean age of $46 \pm 21.2$ years; homicides are more frequents in weekend (Saturday and Sunday) and during October. Toxicological analysis of the victims showed ethanol in $47.4 \%$, benzodiazepines in $14 \%$, cocaine in $12.3 \%$, opium derivatives in $10.5 \%$ and tetrahydrocannabinol in 8.8\%.

A detailed analyses was also carried out in the cases of homicides occurred in the setting of gender violence due to its high prevalence in our country. In 18 out of 19 cases, women died in the hands of her couple, ex-couple, or first-degree relative.

Results have been compared with similar national and international studies.

Key words: homicide, forensic pathology, gender violence.

Correspondencia: Dr. Joaquín Lucena Romero. Servicio de Patología Forense. IML de Sevilla. Avda. Sánchez Pizjuán s/n. 41009 SEVILLA. Tel. 954370644, Fax 954906834. E-mail: iaf.sevilla@andaluciajunta.es

\footnotetext{
1 Servicio de Patología Forense. Instituto de Medicina Legal (Sevilla).

2 Médico forense en prácticas.

3 Servicio de Química. Instituto Nacional de Toxicología y Ciencias Forenses (Sevilla).
} 


\section{INTRODUCCIÓN:}

La palabra homicidio deriva etimológicamente del latín: homo (hombre) y caedere (matar). Es, por lo tanto, el acto por el que una persona quita la vida a otra o le da muerte [I]. La conducta homicida del ser humano no es nueva y existe desde tiempos inmemoriales, siendo un crimen inaceptable para todas las sociedades [2].

El homicidio es siempre un reto para el patólogo forense, quien debe actuar minuciosamente desde el momento de la diligencia de inspección ocular y levantamiento del cadáver en la escena del crimen, así como durante la práctica de la autopsia médico-legal, para ser capaz de reconstruir los hechos y determinar el origen y las circunstancias de la muerte.

El número de homicidios es uno de los parámetros que mejor refleja el grado de seguridad ciudadana de una población por lo que su análisis aporta una valiosa información no solo de interés médico-forense sino también social. [3]

En este trabajo se realiza un estudio epidemiológico descriptivo de los homicidios ocurridos en la provincia de Sevilla desde 2004 hasta 2007 con el objetivo de valorar las características asociadas a los mismos y compararlas con los estudios realizados tanto en el ámbito nacional con internacional.

\section{MATERIAL Y MÉTODOS:}

Se trata de un estudio retrospectivo de las muertes por homicidio ocurridas en la provincia de Sevilla desde el I de enero de 2004 hasta el 3 I de diciembre de 2007 (población de referencia a I de enero de 2007 de 1.849.268 habitantes, de los cuales 907.853 son hombres y 941.415 son mujeres) [4].

Los datos han sido recogidos de la información existente en los archivos del Servicio de Patología Forense del Instituto de Medicina Legal de Sevilla en los que se refleja el resultado de la investigación medico-legal de las muertes homicidas, incluyendo protocolo de levantamiento del cadáver, informe de autopsia y resultado de las pruebas complementarias realizadas por el departamento de Sevilla del Instituto Nacional de Toxicología y Ciencias Forenses.

Durante estos cuatro años, del total de 3632 autopsias realizadas, 57 correspondieron a muertes de etiología homicida (1.6\%). De cada uno de los casos se han obtenido los siguientes datos: edad y sexo de la víctima, mecanismo de la muerte, fecha del homicidio, relación víctimaagresor y resultados de toxicología. Del presente estudio se han excluido aquellas muertes violentas que tras su investigación han sido consideradas como accidentales desde el punto de vista médico-forense, aun cuando jurídicamente puedan ser calificadas como homicidios imprudentes.

A partir de la información disponible, las variables analizadas estadísticamente han sido:

- Tasa de homicidios.

- Distribución por sexos y grupos de edad.

- Presencia de tóxicos en las víctimas.

- Evolución temporal.

- Mecanismo de la muerte.

- Casos relacionados con violencia de género.

\section{RESULTADOS:}

El número de muertes homicidas durante el período de cuatro años estudiado fue de 57 , lo que supone el $1.6 \%$ del total de autopsias (3632) y el $2.9 \%$ de las muertes violentas (1975) (Fig. I), con una tasa media de 0.77 por 100.000 habitantes ( 1.04 por 100.000 en hombres y 0.50 por 100.000 en mujeres). 


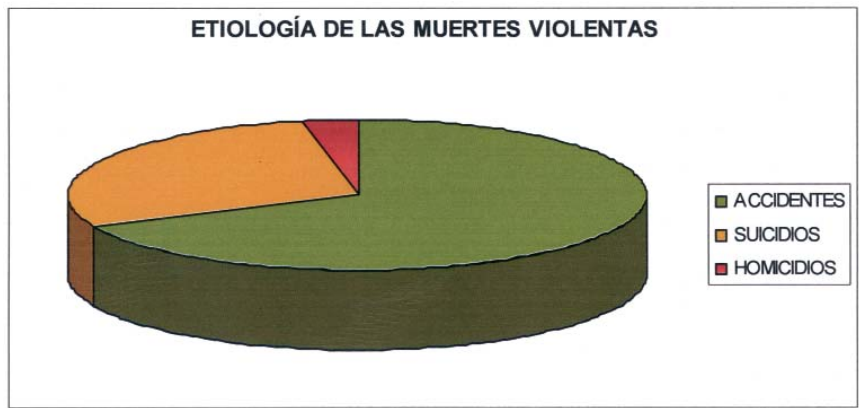

Figura 1. Etiología médico-legal de las muertes violentas durante el período 2004-2007.

En el año 2004, del total de autopsias realizadas (919), el 2. I\% correspondieron a muertes homicidas (19) lo que representa el 3.5\% de las muertes violentas. La tasa de homicidios de este año es de 1.03 por 100.000 habitantes ( 1.43 por I 00.000 en hombres y 0.64 por 100.000 en mujeres).

En el año 2005 el I.2\% ( I I ) de las autopsias totales (92 I) fueron de etiología homicida, lo que supone el $2.4 \%$ de las muertes violentas (456). La tasa de homicidios de este año es de 0.59 por 100.000 habitantes ( 0.88 por 100.000 en hombres y 0.32 por 100.000 en mujeres).

Durante el año 2006 se practicaron un total de 914 autopsias de las cuales el $1.6 \%$ (I5) corresponden a homicidios, suponiendo el $3 \%$ de las muertes violentas (498). La tasa de homicidios de este año es de 0.81 por 100.000 habitantes ( 0.99 por 100.000 en hombres y 0.64 por 100.000 en mujeres).

En el año 2007 se autopsiaron 878 cadáveres y de ellos el I.4\% (I2) fueron homicidios, lo que representa el $2.5 \%$ del total de muertes violentas (479). La tasa de homicidios de este año es de 0.65 por 100.000 habitantes ( 0.88 por 100.000 en hombres y 0.42 por 100.000 en mujeres).

El número de homicidios anuales durante estos cuatro años no ha sufrido grandes variaciones, oscilando entre el máximo de 19 casos ocurridos en 2004 y el mínimo de I I en 2005, lo que supone una media de $14.25 \pm 3.6$ homicidios/año (Fig. 2).

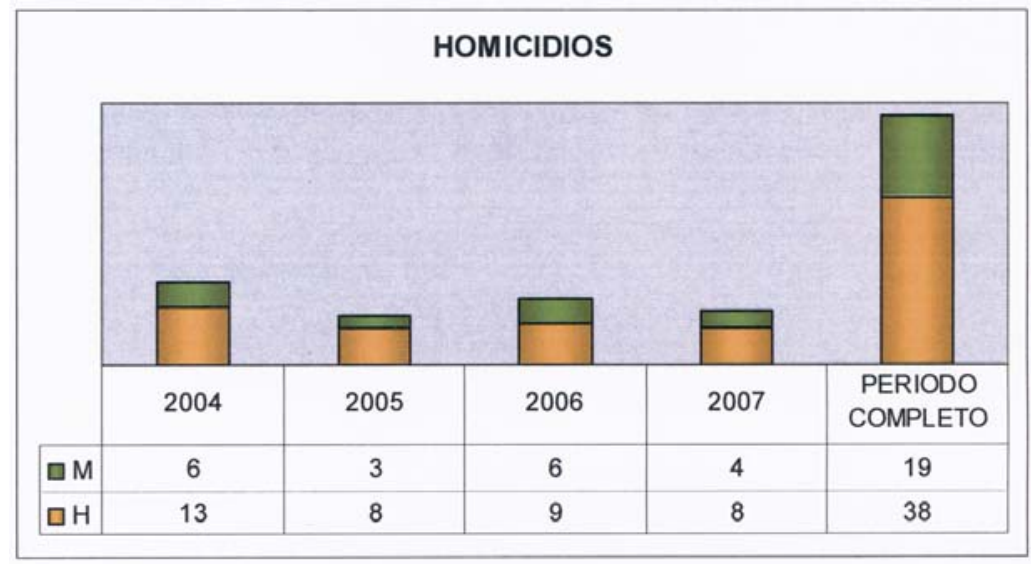

Figura 2. Distribución de los homicidios por año y sexo de la víctima. 
MECANISMO:

Los principales mecanismos empleados para la comisión de los homicidios fueron arma blanca (49.1\%), arma de fuego (21\%), contusiones -incluyendo los traumatismos craneoencefálicos (TCE) y agresión con instrumento contuso- ( $15.8 \%)$, asfixia mecánica -estrangulación y sofocación$(8.8 \%)$ y otros (5.3\%) -entre ellos: quemaduras, envenenamiento y abandono de un menor con parálisis cerebral que fallece por deshidratación- (Fig. 3).

Entre las mujeres, las lesiones por arma blanca son responsables de la muerte en un $52.6 \%$ de los casos, las armas de fuego en el 21\%, seguidas por la asfixia mecánica con un 15.8\% (2 casos de sofocación y I de estrangulación a mano) y otros métodos (sobredosis de insulina y quemaduras) con el $10.5 \%$.

En los homicidios en que las víctimas son hombres, el mecanismo más utilizado es el arma blanca (47.4\%), las contusiones representan un $23.7 \%$ y las armas de fuego un $21 \%$. Las asfixias mecánicas (sofocación) están implicadas en el 5.3\% de los casos y, por último, otros métodos (deshidratación por abandono) representan el 2.6\%.

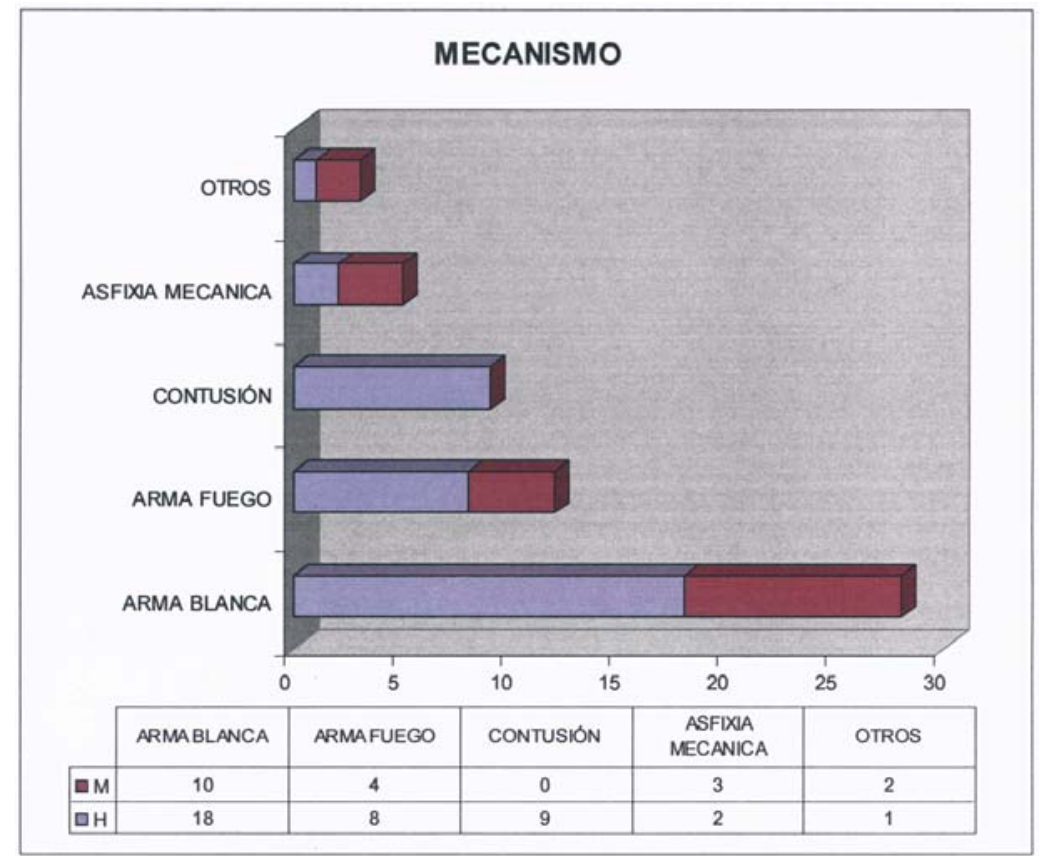

Figura 3. Mecanismos de muerte homicida según el sexo de la víctima.

\section{SEXO Y EDAD DE LA VÍCTIMA:}

De las 57 víctimas de homicidios durante estos cuatro años, el 66.7\% fueron hombres (38) y el 33.3\% mujeres (19), lo que supone una relación hombre/mujer de 2: I.

El pico de mayor incidencia se registra en el grupo de edad de 31 a 40 años ( 16 casos), con el $28.1 \%$ de las víctimas. Por sexos, el mayor número de varones se ubican dentro de esta década ( 12 casos), mientras que en las mujeres el rango de edad más frecuentemente implicado es el de mayores de 80 años (6 casos).

La edad media es $46 \pm 21.2$ años, con un rango de I a 84 años (Fig. 4). 


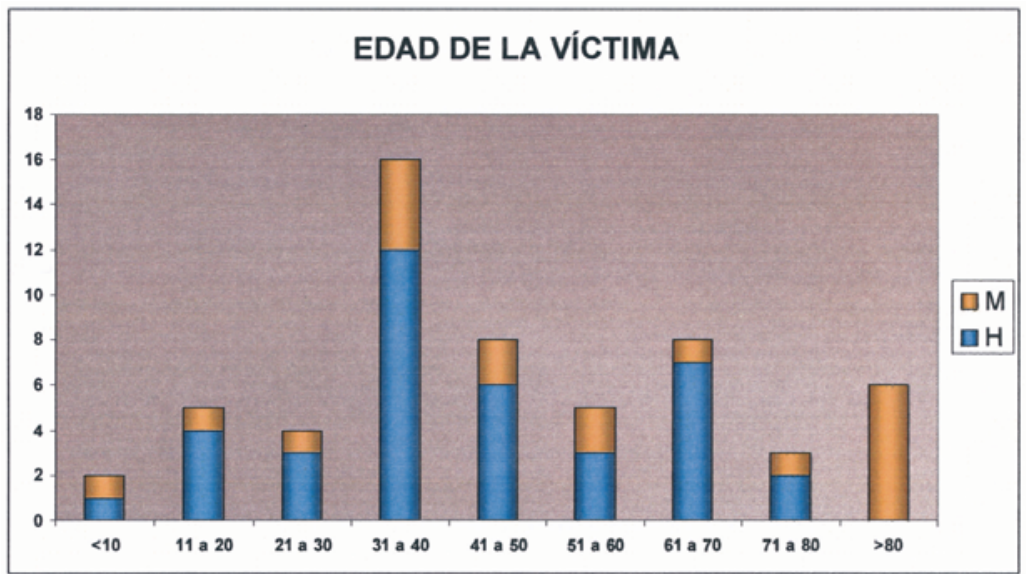

Figura 4. Distribución de los casos de homicidios según edad y sexo de la víctima.

\section{EVOLUCIÓN TEMPORAL:}

Octubre fue el mes en el que sucedieron más homicidios con un total de 9 casos (I5.8\%), frente a enero, marzo, junio, septiembre y diciembre con un número de 3 (5.3\%) en cada uno de ellos.

En cuanto a los días de la semana, el 28.1\% (16) ocurrieron en domingo y sólo un 3.5\% (2) lo hicieron en jueves. Durante el fin de semana -sábado y domingo- tuvieron lugar el $47.4 \%$ de los casos (27); en el sexo femenino el 68.4\% (I3) de los homicidios fueron cometidos en este período de tiempo y en el caso de los hombres lo fueron el 36.8\% (I4) (Fig. 5).

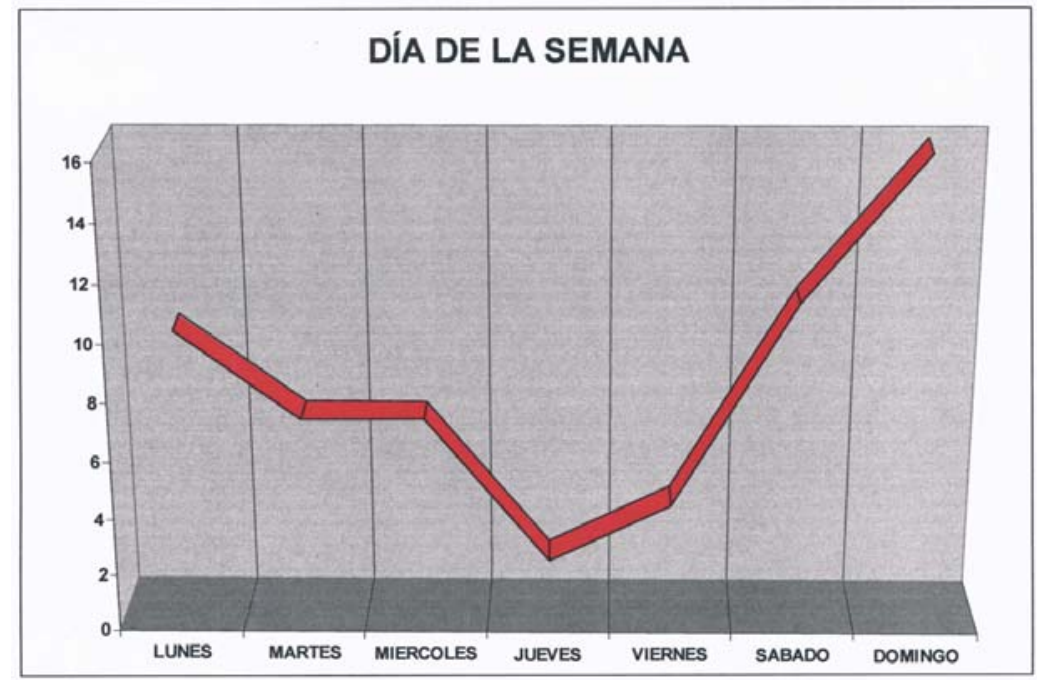

Figura 5. Distribución de los homicidios según el día de la semana.

\section{ANÁLISIS TOXICOLÓGICO:}

Durante la práctica de la autopsia médico-legal se tomaron distintas muestras biológicas para estudios complementarios, entre ellos sangre para análisis toxicológico. En 9 de los 57 casos (I5.8\%) no se realizó análisis toxicológico ya que las víctimas estuvieron hospitalizadas entre el momento de la agresión y el de la muerte, con lo que los resultados no serían valorables. 
En el 47.4\% (27 casos) se detectó etanol en sangre, 20 eran hombres y 7 mujeres, lo que supone que el $52.6 \%$ de las víctimas varones y el $36.8 \%$ de las víctimas mujeres presentaban resultados positivos para etanol.

En el I4\% (8 casos) se obtuvieron resultados positivos para benzodiacepinas. Entre las drogas de abuso, se detectaron cocaína o sus metabolitos en el $12.3 \%$ (7) de las víctimas, opiáceos en el 10.5\% (6) y tetrahidrocannabinol en el $8.8 \%$ (5).

El 22.8\% ( I 3 casos) presentaba positividad para varias de las sustancias mencionadas.

Finalmente, en 15 de ellos (26.3\%) los resultados de toxicología fueron negativos. (Fig. 6).

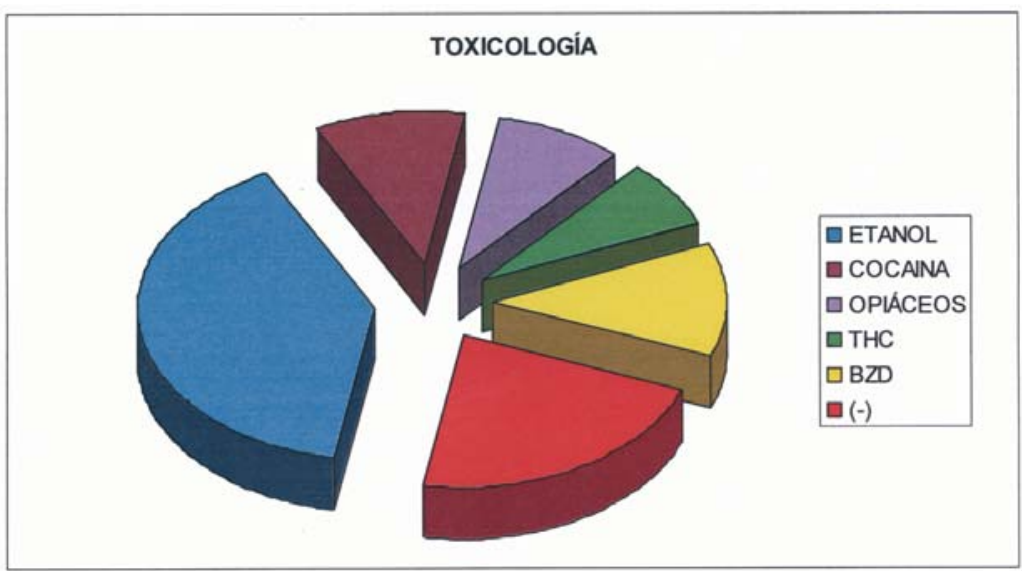

Figura 6. Distribución de los tóxicos encontrados en el análisis toxicológico.

HOMICIDIOS DE MUJERES EN EL ÁMBITO DE VIOLENCIA DOMÉSTICA Y DE GÉNERO:

Del total de los homicidios en que la víctima era mujer, sólo en uno de los casos (5.3\%) el autor no pertenecía al ámbito familiar, mientras que el 94.7\% ( 8 casos) fueron cometidos por familiares directos de la fallecida y, en particular, el 63.2\% ( 12 casos) por sus parejas o ex-parejas sentimentales (Tabla I).

\begin{tabular}{c|ccc}
\hline & Pareja o ex-pareja & Otro familiar & Extraño \\
\hline 2004 & 4 & 2 & 0 \\
2005 & 2 & 1 & 0 \\
2006 & 3 & 3 & 0 \\
2007 & 3 & 0 & 1 \\
\hline TOTAL & 12 & 6 & 1 \\
\hline
\end{tabular}

Tabla 1. Distribución de los homicidios en mujeres según año y relación con el autor.

La tasa media de homicidios de mujeres dentro del seno familiar ha sido de 0.24 por I 00.000 habitantes (rango 0.16-0.32 por 100.000) (Fig. 7).

El arma blanca fue el método usado con más frecuencia en los casos de muerte relacionada con violencia de género con un $66.7 \%$ (8 casos), seguido por el arma de fuego con un $25 \%$ (3 casos) y la asfixia mecánica (sofocación) en un solo caso (8.3\%). 


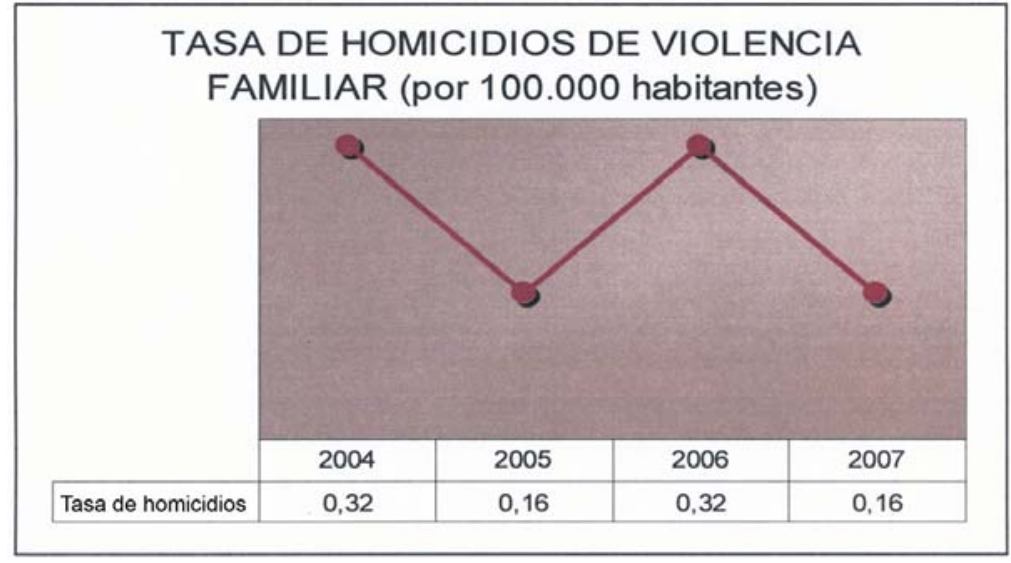

Figura 7. Tasa de homicidios en violencia familiar según el año.

La edad media de las mujeres víctimas de homicidio por violencia de género fue de $49 \pm 23.5$ años, con un pico mayor de incidencia entre los 31-40 (25\%); sin embargo la mayoría de las víctimas de violencia familiar fueron mayores de 80 años (27.8\%).

El 68.4\% de los homicidios en mujeres, independientemente del agresor, acontecieron en fin de semana (sábado y domingo), y en el caso particular de la violencia de género el porcentaje asciende al 75\% (9 de los 12 homicidios).

En 5 de los 18 casos ocurridos en el ámbito doméstico, el autor se suicidó después de cometer el crimen, lo que supone un porcentaje del $27.8 \%$. Uno de los homicidas realizó un intento de suicidio sin consumarlo, si lo incluimos dentro de los comportamientos autolíticos posteriores al homicidio alcanzaríamos cifras del 33.3\% de casos de homicidio-suicidio.

\section{DISCUSIÓN:}

En el presente estudio se valoran las características epidemiológicas de los 57 homicidios ocurridos en la provincia de Sevilla desde el I de enero del 2004 hasta el 3 I de diciembre del 2007.

Durante los cuatro años analizados el número de homicidios se ha mantenido estable, con un mínimo de I I casos en 2005 y un máximo de 19 en 2004, lo que supone una tasa media de homicidios de 0.77 por 100.000 habitantes (rango 0.59-1.03 por 100.000). Diferenciando por sexos, la tasa media de homicidios en hombres es 1.04 por 100.000 y en mujeres 0.50 por 100.000. Si comparamos estos resultados con los publicados en 1990 para la Comunidad Andaluza, donde la tasa para hombres era de 1.35 por 100.000 habitantes y para mujeres de 0.34 por I 00.000 habitantes [5], se observa una ligera disminución en los casos en los que la víctima era varón mientras que en las mujeres se aprecia un aumento.

Atendiendo a las tasas de homicidio recogidas a nivel nacional (0.8 por 100.000 habitantes) obtenemos unos datos afines, igual ocurre con las cifras de Francia (0.8 por 100.000). En los países de nuestro entorno, Reino Unido registra la tasa más baja (0.4 por 100.000) mientras que Portugal e Italia [6] recogen cifras superiores ( 1.7 y 1.98 por 100.000 habitantes respectivamente). En Europa las tasas más altas corresponden a Estonia y Lituania con 8.8 homicidios por 100.000 habitantes y Letonia con 10 por 100.000 [4].

En otros países con diferentes características socio-culturales las tasas de homicidios son más dispares. Así, encontramos datos como los de Japón (0.75-I por 100.000) muy similares a los 
nuestros, o Estados Unidos (6.6 por 100.0000) con cifras más elevadas. Destaca, por ser una de las tasas más altas del mundo, el caso de Sudáfrica (Ciudad del Cabo) con 57 homicidios por 100.000 habitantes [7].

Los datos de los que disponemos muestran que el mecanismo homicida más frecuentemente utilizado es el arma blanca (49. I\%) posiblemente por su mayor disponibilidad. Las armas de fuego ocupan el segundo lugar (21\%). Las contusiones (agresión con objeto contuso y TCE) suponen el 15.8\% y, finalmente, las asfixias mecánicas (estrangulación y sofocación) se emplean sólo en casos esporádicos (8.8\%).

Comparando estos resultados con los estudios dentro del territorio español, las cifras son muy similares a las publicadas en la ciudad de Barcelona (arma blanca 45\%, arma de fuego 30.3\% y contusiones II.3\%) [3].

En estudios internacionales los resultados son más discordantes. Mientras que en Finlandia [8] y Malasia [9] el arma blanca es también el método más empleado (en el 39\% y $41 \%$ respectivamente), en Italia [6], Turquía [ I0], Colombia [ I I] y Estados Unidos [9, I2] lo es el arma de fuego puesto que la legislación sobre su tenencia es menos restrictiva. Sin embargo, en la India [13] las contusiones ocupan el primer lugar siendo responsables del $41 \%$ de las muertes homicidas, seguidas del arma blanca (37.8\%) y las asfixias mecánicas (3.3\%), sin que existan estadísticas significativas de homicidios perpetrados con armas de fuego.

Si valoramos el sexo de las víctimas de los homicidios que estudiamos, el $66.7 \%$ son hombres y el resto (33.3\%) mujeres. Son datos que coinciden con todas las series analizadas. En ambos sexos el mecanismo más utilizado fue el arma blanca; en hombres las contusiones ocupan el segundo lugar seguidas del arma de fuego. Sin embargo, en las mujeres es el arma de fuego el segundo más frecuentemente implicado, no registrándose ningún caso de agresión por mecanismo contuso.

La edad de las víctimas que recogemos se encuentra distribuida entre I y 84 años, con una edad media de $46 \pm 21$.2. En Francia la edad media es de 42 años [7] y en Finlandia de 40.8 para hombres y 40.9 para mujeres [8], resultados muy próximos a los nuestros. El presente estudio refleja también que el grupo de edad más frecuentemente implicado en muertes de etiología homicida es el de 31-40 años, donde se sitúan el 28. I\% de los casos totales. Comparar estos resultados con las series de la bibliografía analizada no siempre es posible puesto que los grupos de edad que se diferencian varían de unas a otras, no obstante, la mayoría manejan datos equivalentes. Así, en Finlandia el pico de mayor incidencia se sitúa entre 31-40 años [8], y en India el 80\% de los casos se encuentra entre los 2 I-50 años con un pico de mayor incidencia entre 31-40 [13]. Otros estudios consideran intervalos de edad más amplios que los nuestros, aún así son resultados equivalentes ya que sus edades más frecuentes incluyen también la tercera década de la vida (Polonia con una mayor incidencia entre 21 -40 años ( 14 ) y Malasia entre 20-39 años [9]). Finalmente, los estudios realizados en Francia muestran resultados diferentes puesto que su pico de incidencia se sitúa entre los 50-59 años [7].

Respecto a la evolución anual de los casos que presentamos, aunque en todos los meses se recoge algún suceso, el pico de incidencia se registra en el mes de octubre con el I5.8\% de los homicidios, circunstancia que coincide con cifras de la India [13], mientras que en Finlandia la mayoría sucedieron durante el mes de julio [8]. A pesar de estos resultados, consideramos que se trata de datos totalmente circunstanciales sin que tengan ninguna relevancia epidemiológica.

Si analizamos la distribución por días de la semana, el domingo es el día en que se cometieron el mayor número de casos con el $28.1 \%$ y si consideramos el fin de semana en su totalidad (sábado y domingo) este porcentaje asciende al 47.4\%. También en la India hay significativamente más homicidios durante el fin de semana (sábado y domingo) [15] y en series de Finlandia hasta un 
tercio de los casos ocurrieron en viernes o sábado [8]. La mayor incidencia de homicidios en fin de semana que se evidencia en los datos expuestos puede estar relacionada con hábitos propios de este período: más tiempo de ocio, relaciones interpersonales más frecuentes e intensas, así como mayor consumo de tóxicos, que predisponen a la aparición de conflictos y comportamientos agresivos e impulsivos.

Los análisis toxicológicos realizados en homicidios suelen centrarse en el autor del delito, sin embargo la presencia de tóxicos en las víctimas puede aportar importante información acerca de las circunstancias en las que se produjeron los hechos. Así, en el $47.4 \%$ de los casos ( 20 hombres y 7 mujeres) se detectó etanol. Otras sustancias halladas fueron cocaína, opiáceos, tetrahidrocannabinol y benzodiacepinas; además, en el 22.8\% de las víctimas se evidenció un consumo concomitante de varias de estas sustancias.

La mayor parte de los artículos revisados centran su valoración en el etanol, solo o acompañado de otras sustancias. Estudios realizados en Finlandia muestran que el 63\% de las víctimas de homicidio habían consumido alcohol previamente, y en particular el 66\% de los hombres y el 39\% de las mujeres [8]. En Lituania las cifras son del $71.2 \%$ en hombres y del $60.3 \%$ en mujeres [16], datos superiores a los obtenidos en nuestro medio donde el etanol estaba presente en el 52.6\% de los hombres y el 36.8\% de las mujeres. En Estados Unidos (Los Ángeles) el $46 \%$ de las víctimas dio positivo a etanol, y diferenciando por sexos el porcentaje en hombres era de $51.3 \%$ y en mujeres de $25.8 \%[17]$.

Como mencionábamos con anterioridad, la mayor incidencia de homicidios durante el fin de semana puede estar relacionada con un mayor consumo de sustancias tóxicas. Nuestros resultados apoyan esta hipótesis, puesto que de las 27 víctimas fallecidas en sábado y domingo, 14 presentaban resultados toxicológicos positivos, lo que representa el $51.8 \%$ de los casos. La mayoría de los autores avalan este hecho y afirman que el consumo de tóxicos, y en particular de etanol, es un factor de riesgo para convertirse en víctima de un delito violento, puesto que exacerba las diferencias interpersonales y aumenta los comportamientos provocativos y la propia vulnerabilidad [ 17 , 18]. Para poder objetivar esta afirmación serían necesarios estudios del consumo de sustancias tóxicas en la población general y en particular de quienes no han sido víctimas de este tipo de delitos.

Merece la pena valorar de forma particular los homicidios en mujeres dentro de los casos que estudiamos y las circunstancias relacionadas con los mismos. Así, resulta especialmente significativo el hecho de que el $94.7 \%$ de ellos ocurran dentro del ámbito familiar, siendo el 63.2\% perpetrado por quienes son o han sido sus cónyuges o por quienes están o han estado ligados a ellas por relaciones similares de afectividad, aun sin convivencia, por lo que deben considerarse homicidios dentro del contexto de Violencia de Género tal y como ésta se define en la Ley Orgánica I/2004, de 28 de diciembre, de "Medidas de Protección Integral contra la Violencia de Género" [19].

La violencia contra las mujeres es un importante problema de salud pública en todo el mundo. Los homicidios en mujeres son un útil indicador de la violencia que sufren las mismas, por el simple hecho de ser mujer, en los distintos ámbitos de su vida, por la situación de desigualdad y discriminación existente tanto en las relaciones personales como sociales; a pesar de lo cual son escasos los estudios médico-legales al respecto y aún menos las comparativas internacionales.

La información referente a los homicidios de mujeres en el ámbito de la violencia doméstica y, posteriormente, también en el ámbito específico de la violencia de género se viene recogiendo en los últimos años por diferentes organismos y entidades, tanto públicas como privadas. Pero no siempre los datos procedentes de las distintas fuentes disponibles son coincidentes, puesto que los criterios utilizados son diversos [20]. 
En la provincia de Sevilla durante estos 4 años se registra una tasa media de homicidios por violencia doméstica y de género de 0.24 por 100.000 habitantes y de 0.48 por 100.000 mujeres, con una evolución anual similar a la del total de Andalucía en la que los años 2004 y 2006 recogen tasas más altas respecto a 2005 y 2007 [20]. En el caso particular de las muertes por violencia de género, la tasa es algo inferior con un valor medio de 0.16 por 100.000 habitantes y de 0.32 por 100.000 mujeres.

Frente a nuestras estadísticas (63.2\%), otros países como Francia (París) recogen que en el $31 \%$ de los homicidios cuya víctima es mujer el autor fue el esposo y en el 20\% el compañero sexual [2 I], en el Reino Unido el 37\% de los homicidios de mujeres fueron cometidos por su pareja o expareja [22]; en el caso de Estados Unidos los homicidios de mujeres dentro de la pareja representan el 30\% [23] y en la India el porcentaje alcanza el $51 \%$ [2]. Por tanto, nuestros datos son superiores, más aún si tenemos en cuenta que el $31.6 \%$ restante fue cometido por otro familiar (madre, padre, hijo o hermano), y que sólo en el 5.3\% (I caso) el agresor fue un extraño.

En nuestro medio el arma blanca fue el método usado con más frecuencia en los casos de muerte relacionada con violencia de género con un $66.7 \%$ (8 casos), seguido por el arma de fuego con un 25\% [3] y finalmente la asfixia mecánica (sofocación) en un solo caso (8.3\%). No se ha encontrado ningún caso de muerte por instrumento contuso, mientras que en las cifras nacionales para 2004 y 2005 recogidos en el Informe anual del Observatorio Estatal de Violencia sobre la mujer fue el segundo mecanismo más utilizado tras el arma blanca [20].

Nuestros resultados coinciden con los datos de Reino Unido y Gales donde el arma blanca y la estrangulación fueron los métodos más usados [22], frente a estudios similares de EE.UU. donde se refleja que el $52.9 \%$ de los casos se cometieron con arma de fuego, el $26.5 \%$ con arma blanca, el $17.7 \%$ por contusiones y solo el $2.9 \%$ mediante asfixia mecánica [23].

El uso preferente del arma blanca en nuestro ámbito y en todo el territorio nacional (estando presente en el 47\% de los homicidios de violencia de género comprendidos entre 1997 y 2004) probablemente tenga relación directa con su mayor disponibilidad, si bien algunos autores consideran que guarda relación con la propia naturaleza del crimen y la furia extrema con la que es cometido (*). Lo que es cierto es que en España existe en la población un menor número de armas de fuego, fundamentalmente en las zonas urbanas, por ello el arma blanca es la más frecuente, ya que existe en todos los hogares. Aún así, las armas de fuego se han utilizado con una gran frecuencia cuando el agresor las empleaba habitualmente bien por su profesión (militares, guardia civil, guardas jurados) o bien por entretenimiento, como los cazadores en zonas rurales [25].

La edad media de las mujeres víctimas de homicidio por violencia de género durante estos cuatro años fue de $49 \pm 23.5$ años. Todas ellas son mayores de edad, recogiendo algún caso en todas las décadas de la vida desde los 18 hasta los 84 años, con una mayor incidencia entre los 3 I-40 (25\%), dato que coincide con las cifras nacionales [20]. Aunque las víctimas de violencia de género son en su mayoría menores de 50 años, si ampliamos el espectro a las víctimas de violencia familiar de forma global llama la atención que son las mujeres mayores de 80 años las más implicadas (27.8\%). A la luz de estos resultados, se pone de manifiesto la enorme heterogeneidad de las víctimas de violencia doméstica y de género, que solo tienen en común el hecho de ser mujeres.

Como ya señalan algunos autores [25], los datos estadísticos no muestran que los casos de homicidios en el contexto de violencia de género se produzcan con mayor frecuencia en unos

(*) Declaraciones de Miguel Lorente, actualmente Delegado del Gobierno de Violencia de Género, al periódico El País en fecha 4 de febrero de 2008 (pág. 38). 
meses del año con respecto a otros. Aunque también se ha señalado que no se puede afirmar que en los fines de semana ocurran más homicidios, nuestros casos sí resultan representativos en este sentido: el 68.4\% de los homicidios en mujeres, independientemente del agresor, acontecieron en fin de semana (sábado y domingo), y en el caso particular de la violencia de género el porcentaje asciende al 75\% (9 de los 12 homicidios). El motivo por el cual se produce esta agrupación de casos durante el fin de semana puede deberse, al igual que en el resto de homicidios, a un mayor tiempo de convivencia que agrava las tensiones ya existentes en la pareja y al incremento del consumo de tóxicos.

En algunas ocasiones, el homicidio se sigue de un comportamiento suicida del autor. En nuestro estudio, este hecho se produce en 6 de los 18 casos (33.3\%) de homicidio dentro del ámbito familiar, y concretamente en 4 de violencia de género (33.3\%). A nivel nacional, el Observatorio Estatal de violencia sobre la mujer recoge que en 2004 y 2005 el suicidio se produjo en el 19.7\% de los casos y en el 2006 en el 25\%. La posición social y el grado de integración del agresor en su entorno son elementos fundamentales para entender estas conductas. De este modo, el suicidio suele ser más frecuente en aquellos homicidas que están muy integrados y que temen ser rechazados por aquellas personas o entornos que son importantes para ellos [20].

A pesar de que la violencia de género con resultado de muerte ocurre desde tiempos inmemoriales y es a menudo el trágico punto final de una relación problemática, son escasos los estudios estadísticos al respecto y los disponibles utilizan criterios de selección y clasificación diferentes unos de otros, lo que limita la posibilidad de establecer comparativas de nuestros resultados con cifras tanto nacionales como internacionales.

Los resultados del presente estudio muestran la magnitud del problema de la violencia contra la mujer en su forma más extrema: la muerte a manos de quien era o había sido su compañero sentimental. Por tanto, debe tomarse como referencia para llevar a cabo una actuación integral sobre esta lacra social.

\section{CONCLUSIONES:}

I. La tasa media de homicidios en la provincia de Sevilla es considerablemente baja, lo que refleja que los delitos de sangre en nuestro medio suceden con escasa frecuencia.

2. El mecanismo más utilizado es el arma blanca, al igual que ocurre en otros países donde la legislación sobre tenencia de armas de fuego es muy restrictiva.

3. Las víctimas son habitualmente varones con una edad media de $46 \pm 21.2$ años.

4. Los fines de semana (sábado y domingo) registran el mayor número de casos, asociados habitualmente a un mayor consumo de tóxicos, principalmente etanol.

5. El $94.7 \%$ de las víctimas mujeres murieron a manos de su pareja, ex-pareja o familiar de primer grado. Esto pone de manifiesto la grave problemática social que existe en relación a la violencia contra la mujer y, en particular, a los homicidios derivados de la misma, que se producen casi de forma exclusiva dentro del ámbito familiar.

\section{AGRADECIMIENTOS:}

A todo el personal administrativo y auxiliar del Servicio de Patología Forense de Sevilla, sin cuya encomiable labor no habría sido posible la elaboración de este artículo. 


\section{BIBLIOGRAFÍA:}

I. Castellano M. Las lesiones en el Código Penal. En Gisbert JA. “Medicina Legal y Toxicología”. 6a ed. Ed. Masson, Barcelona 2004. pp 309-321.

2. Mohanty MK, Panigrahi MK, Mohanty S, Das SK. Victimiologic study of female homicide. Legal Med 2004;6:15I-56.

3. Dolado J, Lucena J, Tortosa T. Evolución de las muertes por homicidio en la ciudad de Barcelona. Período 1982-1996. Quartes jornades catalanes d'actualització en Medicina Forense. Ed. Generalitat de Catalunya, Departament de Justicia. Barcelona 1999.

4. Instituto Nacional de Estadística. Defunciones según la causa de muerte. Defunciones por Comunidad Autónoma de residencia, causas, sexo y edad. Tasas estandarizadas de mortalidad por países, causa de muerte, período y sexo. www.ine.es

5. Bernal M, Gómez EM, Gutiérrez E, Lafita S, Guillén C. Estudio de suicidios y homicidios en España. Años 1980-1990. Actas Luso-Esp Neurol Psiquiatr 1995;23:249-255.

6. Preti A, Miotto P. Death by homicide in Italy, 1980-94: age and gender differences among victims. Med Sci Law 2000;40:233-40.

7. Saint-Martin P, Bouyssy M, Bathellier S, Sarraj S, O'Byrne P. Homicide in Tours (Indre-et-Loire, France): A four-year review. J Clin Forensic Med 2006;13:331-34.

8. Wahlsten P, Koiranen V, Saukko P. Survey of medico-legal investigation of homicides in the city of Turku, Finland. J Clin Forensic Med 2007;14:243-252.

9. Kumar V, Li AK, Zanial AZ, Lee DA, Salleh SA. A study of homicidal deaths in medico-legal autopsies at UMMC, Kuala Lumpur. J Clin Forensic Med 2005;12:254-57.

I0. Hilal A, Cekin N, Gülmen MK, Ozdemir MH, Karanfil R. Homicide in Adana, Turkey: a 5-year review. Am J Forensic Med Pathol 2005;26:14I-45.

II. Cardona M, García HI, Giraldo CA, López MV, Suárez CM, Corcho DC, Posada CH, Flórez MN. Homicides in Medellín, Colombia from 1990 to 2002: victims, motives and circumstances. Cad Saude Publica 2005;21:840-51.

I2. Arbuckle J, Olson L, Howard M, Brillman J, Anctil C, Sklar D. Safe at home? Domestic violence and other homicides among women in New México. Ann Emerg Med 1996;27:210-15.
13. Ambade VN, Godbole HV, Kukde HG. Suicidal and homicidal deaths: a comparative and circumstantial approach. J Forensic Leg Med 2007;:14:253-60.

14. Rygol K, Chowaniec C, Kobek M, Chowaniec M. Forensic analysis of homicides on the basis of cases examined in the Forensic Medicine Department, Medical University of Silesia, Katowice, in the years 1991-2002. Forensic Sci Int 2005;|147 Suppl:S75-6.

15. Mohanty MK, Kumar TS, Mohanram A, Palimar V. Victims of homicidial deaths - an análisis of variables. J Clin Forensic Med 2005; 12:302-4.

16. Benosis A, Rybalko ]. Alcohol consumption in homicide victims. Forensic Sci Int 2007; 169 Suppl I: SI8.

17. Goodman RA, Mercy JA, Loya F, Rosenberg ML, Smith JC, Allen NH, Vargas L, Kolts R. Alcohol use and interpersonal violence: alcohol detected in homicide victims. AJPH 1986;76:144-49.

18. Muscat JE, Huncharek MS. Firearms and adult, Domestic Homicides. The role of alcohol and the victim. Am J Forensic Med Pathol 1991;12:105-110.

19. Ley Orgánica I/2004, de 28 de diciembre, de "Medidas de Protección Integral contra la Violencia de Género". BOE n. 313 de 29/12/2004, páginas 42166-42197.

20. Observatorio Estatal de Violencia sobre la Mujer. Informe Anual del Observatorio Estatal de Violencia sobre la Mujer 2007. Informe sobre muertes violentas en el ámbito de la violencia doméstica y de género 2007. www.observatorioviolencia.org

2I. Lecomte D, Tucker ML, Fornes P. Homicide in women: a report of 44I cases from Paris and its suburbs over a 7-year period. Sixth Cross Channel Conference on Forensic Medicine 1998.

22. Aldridge ML, Browne KD. Perpetrators of spousal homicide: a review. Trauma Violence Abuse 2003;4:265-76.

23. Wadman MC, Muelleman RL. Domestic violence homicides: ED use before victimization. Am J Emerg Med 1999;17:689-91.

24. Genovés $A$. La violencia de género mata. Estudio sobre la violencia de género con resultado de muerte, análisis sistemático de las sentencias y datos estadísticos. Ed.:Instituto Andaluz de la Mujer 2007. 